Research Article

\title{
Flip and Neimark-Sacker Bifurcations in a Coupled Logistic Map System
}

\author{
A. Mareno ${ }^{1}{ }^{1}$ and L. Q. English ${ }^{2}$ \\ ${ }^{1}$ Department of Mathematics and Computer Science, Pennsylvania State University, Capital College, Middletown, \\ PA 17057, USA \\ ${ }^{2}$ Department of Physics, Dickinson College, Carlisle, PA 17013, USA
}

Correspondence should be addressed to A. Mareno; aum24@psu.edu

Received 18 April 2020; Accepted 19 June 2020; Published 16 July 2020

Academic Editor: Luca Pancioni

Copyright (c) 2020 A. Mareno and L. Q. English. This is an open access article distributed under the Creative Commons Attribution License, which permits unrestricted use, distribution, and reproduction in any medium, provided the original work is properly cited.

In this paper, we consider a system of strongly coupled logistic maps involving two parameters. We classify and investigate the stability of its fixed points. A local bifurcation analysis of the system using center manifold theory is undertaken and then supported by numerical computations. This reveals the existence of a flip and Neimark-Sacker bifurcations.

\section{Introduction}

Coupled logistic maps originally gained attention in the mathematical biology literature via their utility in models of, for instance, populations of migrating species and environmental heterogeneity [1-4]. Recent years, however, have seen a renewed interest in the dynamics of coupled logistic maps. At least two developments have spurred this re-examination: (a) the realization that discrete coupled maps could be usefully exploited in digital encryption schemes [5-7] and (b) success with their experimental implementation using electronic circuits [8-10]. Both of these recent threads have revealed intricate and nonintuitive behavior of these coupled maps.

One such behavior-spontaneous symmetry breaking-was recently highlighted and explored in [8]. That reference, however, did not attempt to analyze the chaotic regime in this coupled system (i.e., for large values of $r$ ), focusing primarily on symmetry breaking and its basin of attraction pertaining to $n$-cycles. What was observed therein was that, as the coupling strength increased, an $n$-cycle would abruptly give way to the symmetry-broken state (also depending on the initial conditions used). No attempt was made to classify this bifurcation. This transition is an interesting phenomenon also seen previously in experiments [10].
Here, we revisit the problem in a mathematically rigorous way and prove that this particular bifurcation is a flip bifurcation $[11,12]$.

For larger $r$-values, beyond those explored in [8], another bifurcation can be seen at even larger coupling values. This was first discovered experimentally in [10], where it was shown that the symmetry-broken state itself undergoes a transition to chaos. This transition, however, did not appear to follow the standard period-doubling route to chaos, and no rigorous attempt was made in that reference to analyze this bifurcation, although it was reasonably speculated in [10] to involve a Neimark-Sacker bifurcation. In this paper, we shed further light on the origins of this bifurcation manifesting for sufficiently large $r$-values and prove that the initial instability is indeed due to a Neimark-Sacker bifurcation.

Throughout this work, we consider the following discrete system:

$$
\begin{aligned}
& x_{n+1}=(1-\epsilon) f\left(x_{n}\right)+\epsilon f\left(y_{n}\right), \\
& y_{n+1}=\epsilon f\left(x_{n}\right)+(1-\epsilon) f\left(y_{n}\right),
\end{aligned}
$$

where

$$
f(z)=r z(1-z) .
$$


For convenience, the system can be rewritten in the form $F(x, y)=((1-\epsilon) f(x)+\epsilon f(y), \epsilon f(x)+(1-\epsilon) f(y))$,

where the parameters $\epsilon \in[0,1]$ and $r \in(0,4)$.

The structure of the paper is as follows. After providing some technical background in Section 2, we start by systematically classifying all the fixed points and their stability properties-in the $r \epsilon$ plane-that manifest in this coupled system in Section 3. In Section 4, we then focus on the symmetry-broken 1-cycle-a fixed point unique to the coupled system - and proceed to apply the center-manifoldtheoretic framework to prove that it becomes stable via a flip bifurcation as the coupling strength parameter is increased. We take the coupling strength, $\epsilon$, to be our bifurcation parameter and not the growth rate, $r$, which is typically chosen. As mentioned, for even higher values of $\epsilon$ and $r$, the symmetry-broken 1-cycle loses stability again. In this context, we prove using standard theorems that the origin of this instability is a Neimark-Sacker bifurcation. In Section 5, we explore the flip and Neimark-Sacker bifurcations numerically and see excellent agreement with the predictions derived from the theorems established in Section 4.

\section{Invariant Manifolds and Center Manifold Theory}

We begin by stating important terminology and concepts relevant to this work (see, for instance, $[13,14]$ ). Generally, we can say that a set $S$ is an invariant set if iterates of the map for any element of $S$ stay in $S$ for all integers. We will loosely think of an invariant manifold as a set which locally has the structure of Euclidean space, typically as surfaces imbedded in $\mathbb{R}^{n}$, for which the function representing the surface has a maximal rank and can therefore be locally represented as a graph, by way of applying the implicit function theorem.

We now define three important linear subspaces, relevant to the study of dynamical systems, spanned by the (generalized) eigenvectors of the Jacobian matrix $D F(x, y)$ at a fixed point $(x, y): E^{\mathrm{s}}$ (the stable subspace), $E^{\mathrm{u}}$ (the unstable subspace), and $E^{c}$ (the center subspace). The associated eigenvalues of each subspace have modulus less than one, greater than one, or equal to one, respectively. When $D F(x, y)$ has no eigenvalues of unit modulus, $(x, y)$ is called a hyperbolic point and so its stability is determined entirely by the eigenvalues themselves. Furthermore, for hyperbolic points, $E^{\mathrm{c}}$ does not exist.

A hyperbolic fixed point is called a sink if the eigenvalues of the Jacobian matrix evaluated at the fixed point have magnitude less than one. Such a fixed point is locally asymptotically stable. If the magnitudes of both eigenvalues are greater than one, the hyperbolic fixed point is called a source and is locally asymptotically unstable. Moreover, a hyperbolic fixed point is called a saddle point if only one of the eigenvalues has magnitude greater than one.

The stable manifold theorem [15] guarantees the existence of local stable and unstable invariant manifolds $W_{\text {loc }}^{\mathrm{s}}$ and $W_{\text {loc }}^{\mathrm{u}}$ which can be viewed as nonlinear analogues of the linear subspaces $E^{\mathrm{s}}$ and $E^{\mathrm{u}}$, respectively. These invariant manifolds are tangent to these the two linear subspaces, have the same dimensions as these subspaces, and are as smooth as the underlying map.

The center manifold theorem (see chapter 1 in [15] or [13]) asserts the existence of an invariant manifold tangent to the center eigenspace $E^{\mathcal{C}}$ which can be nonunique and "nonsmooth" (in a certain sense) (see chapter 3 in [15] or [13]), where the dynamics of the nonlinear system (at, say, the trivial fixed point) restricted to the center manifold is determined by a $c$-dimensional map, a map whose dimension is the same as that of the center subspace $E^{c}$, where $(x, y) \in \mathbb{R}^{c} \times \mathbb{R}^{s}$ and both $\mathbb{R}^{s}$ and $\mathbb{R}^{c}$ are subsets of $\mathbb{R}^{n}$. So, for a two-dimensional system, such as the system studied in this paper, the dynamics of the nonlinear map are determined by a one-dimensional map.

Herein lies the significance of center manifold theorem, rather than studying the map on the entire domain of the map to determine its dynamics, in which we can restrict this analysis to the center manifold, an invariant manifold with dimension equal to the dimension of the center subspace, which is less than the dimension of the map's domain. In addition, using the invariance of the center manifold one can derive a quasi-linear partial differential equation that the $c$ dimensional map characterizing the center manifold must satisfy in order for its graph to be an invariant center manifold. To find this map, one must solve this partial differential equation. Thus, this theorem can be viewed as type of reduction principle that one can apply to ascertain the stability of nonhyperbolic fixed points when $E^{\mathrm{u}}$ is trivial.

Therefore, in this paper, we restrict our use of center manifold theory to the case where the Jacobian matrix has its spectrum inside the unit circle apart from one or two eigenvalues. For an additional reference on center manifold theory, see [16].

\section{Classification of the Fixed Points of the Nonlinear System}

We begin our analysis of system (3) by solving the equations

$$
\begin{aligned}
& (1-\epsilon) r x(1-x)+\epsilon r y(1-y)=x, \\
& \epsilon r x(1-x)+(1-\epsilon) r y(1-y)=y
\end{aligned}
$$

and obtaining the fixed points of our system, as shown in more detail in [8]:

$(0,0)$

$$
\begin{array}{r}
\left(\frac{r}{r-1}, \frac{r}{r-1}\right), \\
\left(x^{*}, y^{*}\right), \\
\left(y^{*}, x^{*}\right),
\end{array}
$$

where 


$$
\begin{aligned}
& x^{*}=\frac{r(2 \epsilon-1)+1-\sqrt{(r(1-2 \epsilon)-1)(r(1-2 \epsilon)+4 \epsilon-1)}}{2 r(2 \epsilon-1)}, \\
& y^{*}=\frac{r(2 \epsilon-1)+1+\sqrt{(r(1-2 \epsilon)-1)(r(1-2 \epsilon)+4 \epsilon-1)}}{2 r(2 \epsilon-1)} .
\end{aligned}
$$

We note that $x^{*}$ and $y^{*}$ are real valued if and only if $\Delta=(1-4 \epsilon)(r-1)^{2}+4 \epsilon^{2} r(r-2) \geq 0$. This occurs when

$$
\begin{gathered}
r \in(3,4), \\
\epsilon \in\left[0, \frac{r-1}{2 r}\right], \\
\text { or } \epsilon \in\left[\frac{r-1}{2(r-2)}, 1\right] .
\end{gathered}
$$

In addition, $x^{*}=y^{*}$ if and only if $\Delta=0$ which occurs when $\epsilon=((r-1) / 2 r)$ or $\epsilon=((r-1) / 2(r-2))$, and so for these values of $\epsilon$ the fixed point $\left(x^{*}, y^{*}\right)$ coincides with one of the two symmetric fixed points: $(0,0)$ or $(((r-1) / r),((r-1) / r))$, respectively. Throughout this work, we consider only $\left(x^{*}, y^{*}\right)$ and not $\left(y^{*}, x^{*}\right)$, its flipped counterpart. Following [8], we determine conditions for a fixed point to be classified as a hyperbolic or nonhyperbolic fixed point, and to determine the stability type of hyperbolic fixed points, we compute the Jacobian of our map $F$ :

$$
D F(x, y)=\left(\begin{array}{cc}
(1-\epsilon) r(1-2 x) & \epsilon r(1-2 y) \\
\epsilon r(1-2 x) & (1-\epsilon) r(1-2 y)
\end{array}\right) .
$$

By solving the characteristic equation,

$$
\operatorname{det}(D F(x, y)-\lambda I)=0 .
$$

The eigenvalues of the Jacobian evaluated at a fixed point $(x, y)$ are computed as follows:

$$
\begin{aligned}
\lambda_{1,2}= & r(1-\epsilon)+r(\epsilon-1)(x+y) \\
& \pm \sqrt{r^{2}(1-2 \epsilon)(x-y)^{2}+\epsilon^{2}(x+y-1)^{2}} .
\end{aligned}
$$

Although the characteristic equation is characterized by the three principle invariants, where each in turn is a function of the eigenvalues of the Jacobian and one can use the Jury conditions to determine the stability of the fixed points, we take a more straightforward approach and analyze the eigenvalues and their magnitudes directly. This direct approach yields more "directional" information about the magnitudes of both eigenvalues.

Using these definitions and the eigenvalues associated with each fixed point, we determine the parameter-dependent regions where each of the fixed points is asymptotically stable, unstable, a saddle point, and a nonhyperbolic point, as stated in the following theorem:

\section{Theorem 1. Fixed point classification and stability}

A. (i) The fixed point $(0,0)$ is sink if $r \in(0,1)$ and $\epsilon \in[0,1]$. (ii) $(0,0)$ is a source if $r \in(0,1)$ and $\epsilon \in[0,((r-1) / 2 r))$ or $\epsilon \in(((r+1) / 2 r), 1]$.

(iii) $(0,0)$ is a saddle point $r \in(1,4)$ and $\epsilon \in(((r-1) / 2 r),((r+1) / 2 r)) \quad$ (here, $\left|\lambda_{1}\right|>1$ and $\left.\left|\lambda_{2}\right|<1\right)$.

(iv) $(0,0)$ is a nonhyperbolic point (specifically here, $\lambda_{2}=-1$ and $\left|\lambda_{1}\right|>1$ ) if

$r \in(1,4)$

$\epsilon=\frac{r+1}{2 r}$

$\lambda_{2}=1,\left|\lambda_{1}\right|>1, \quad$ for $r \in(1,4), \epsilon=\frac{r-1}{2 r}$,

$\lambda_{1}=1, \lambda_{2}=-1, \quad$ for $\epsilon=1, r=1$,

$\lambda_{1}=1,\left|\lambda_{2}\right|<1, \quad$ for $\epsilon \in(0,1), r=1$,

$\lambda_{1}=\lambda_{2}=1, \quad$ for $\epsilon=0, r=1$ (1: 1 resonance $)$.

B. (i) The symmetric fixed point $(((r-1) / r),((r-1) / r))$ is a sink if

$r \in(1,3), \quad$ for all $\epsilon$ in $[0,1]$.

(ii) $(((r-1) / r),((r-1) / r))$ is a source if

$$
r \in(0,1) \text { and } \epsilon \in\left[0, \frac{r-1}{2(r-2)}\right)
$$$$
\text { or } r \in(0,1) \text { and } \epsilon \in\left(\frac{r-3}{2(r-2)}, 1\right] \text {, }
$$$$
\text { or } r \in(3,4) \text { and } \epsilon \in\left[0, \frac{r-3}{2(r-2)}\right) \text {, }
$$

or $r \in(3,4)$ and $\epsilon \in\left(\frac{r-1}{2(r-2)}, 1\right]$.

(iii) $(((r-1) / r),((r-1) / r))$ is a saddle point (in this case it means $\left|\lambda_{1}\right|<1$ and $\left.\left|\lambda_{2}\right|>1\right)$ if

$$
r \in(0,1) \text { and } \epsilon \in\left(\frac{r-1}{2(r-2)}, \frac{r-3}{2(r-2)}\right)
$$$$
\text { or } r \in(3,4) \text { and } \epsilon \in\left(\frac{r-3}{2(r-2)}, \frac{r-1}{2(r-2)}\right) \text {. }
$$

(iv) $(((r-1) / r),((r-1) / r))$ is a nonhyperbolic point if 
$r \in(0,1)$ or $r \in(3,4)$ and $\epsilon=\frac{r-3}{2(r-2)}$,

$$
\text { (here } \left.\lambda_{1}=-1,\left|\lambda_{2}\right|>1\right) \text {, }
$$

or $\epsilon \in(0,1)$ and $r=3, \quad\left(\right.$ here $\left.\lambda_{2}=-1,\left|\lambda_{1}\right|<1\right)$,

or $\epsilon \in(0,1)$ and $r=1, \quad\left(\right.$ here $\left.\lambda_{2}=1,\left|\lambda_{1}\right|<1\right)$,

$$
\begin{gathered}
\text { or } \epsilon=\frac{r-1}{2(r-2)} \text { and } r \in(0,1) \text { or } r \in(3,4) \text { and } \\
\\
\lambda_{1}=1,\left|\lambda_{2}\right|>1
\end{gathered}
$$

\section{Furthermore,}

$\lambda_{1}=\lambda_{2}=-1, \epsilon=0, r=3,(1: 2$ resonance $)$

$\lambda_{1}=1, \lambda_{2}=-1, \epsilon=1, r=3$.

C. (i) The nonsymmetric fixed point $\left(x^{*}, y^{*}\right)$ is a sink if

$r \in(3,1+\sqrt{6})$ and $\epsilon \in\left(\frac{1}{2}+\frac{\sqrt{3}}{2} \sqrt{\frac{1}{r(r-2)}}, 1\right]$,

or $r \in(1+\sqrt{6}, 4)$ and $\epsilon \in\left(\frac{1}{2}+\frac{\sqrt{3}}{2} \sqrt{\frac{1}{r(r-2)}}, f_{2}(r)\right)$,

where

$f_{2}(r)=\frac{1}{4}\left[\frac{3-4 r+2 r^{2}}{r(r-2)}+\frac{\sqrt{9-16 r+8 r^{2}}}{r^{2}(r-2)^{2}}\right]$.

(ii) $\left(x^{*}, y^{*}\right)$ is a source if

$$
r \in(3,4) \text { and } \epsilon \in\left[0, \frac{1}{2}-\frac{\sqrt{3}}{2} \sqrt{\frac{1}{r(r-2)}}\right),
$$

or $r \in(1+\sqrt{6}, 4)$ and $\epsilon \in\left(f_{2}(r), 1\right]$.

(iii) $\left(x^{*}, y^{*}\right)$ is a saddle point if $r \in(3,4)$ and

$$
\begin{array}{r}
\epsilon \in\left(\frac{r-1}{2(r-2)}, \frac{1}{2}+\frac{\sqrt{3}}{2} \sqrt{\frac{1}{r(r-2)}}\right], \\
\left(\text { specifically }\left|\lambda_{2}\right|<1,\left|\lambda_{1}\right|>1\right),
\end{array}
$$

or $r \in[3,4)$ and $\epsilon \in\left(\frac{1}{2}-\frac{\sqrt{3}}{2} \sqrt{\frac{1}{r(r-2)}}, \frac{r-1}{2 r}\right)$,

$$
\text { (specifically }\left|\lambda_{1}\right|<1,\left|\lambda_{2}\right|>1 \text { ). }
$$

(iv) $\left(x^{*}, y^{*}\right)$ is a nonhyperbolic point if

$$
\begin{aligned}
& r \in[1+\sqrt{6}, 4), \\
& \epsilon=f_{2}(r),
\end{aligned}
$$

(here $\left.\left|\lambda_{1}\right|=\left|\lambda_{2}\right|=1, \lambda_{i} \in \mathbb{C}, i=1,2\right)$ or

$$
\begin{gathered}
r \in(3,4) \text { and } \epsilon=\frac{1}{2}+\frac{\sqrt{3}}{2} \sqrt{\frac{1}{r(r-2)},} \\
\left(\text { specifically, } \lambda_{2}=-1,\left|\lambda_{1}\right|<1\right), \\
\text { or } r \in(3,4) \text { and } \epsilon=\frac{1}{2}-\frac{\sqrt{3}}{2} \sqrt{\frac{1}{r(r-2)},} \\
\text { (specifically, } \left.\lambda_{2}=-1,\left|\lambda_{1}\right|>1\right),
\end{gathered}
$$

or $r=3$ and $\epsilon=0$, where our system now corresponds to an uncoupled pair of logistic maps.

Proof. For the trivial fixed point $(0,0), \lambda_{1}=r$ and $\lambda_{2}=$ $r(1-2 \epsilon)$. By inspection, we see $\left|\lambda_{i}\right|<1$, for $i=1,2$ if and only if $r \in(0,1)$ for any epsilon in $[0,1]$. The remaining parts of A can easily be deduced.

For the symmetric fixed point $(((r-1) / r),((r-1) / r))$, $\lambda_{1}=r(1-2 \epsilon)((2 / r)-1)$ and $\lambda_{2}=r((2 / r)-1)$. Again a straightforward calculation shows that parts (i)-(iv) of B hold.

For the antisymmetric fixed point $\left(x^{*}, y^{*}\right)$, a direct calculation shows that the eigenvalues are

$$
\begin{aligned}
& \lambda_{1}=\frac{\epsilon-1+(2 \epsilon-1) \sqrt{\left(\epsilon^{2}+(1-2 \epsilon) \Delta\right) /(1-2 \epsilon)^{2}}}{2 \epsilon-1}, \\
& \lambda_{2}=\frac{\epsilon-1+(1-2 \epsilon) \sqrt{\left(\epsilon^{2}+(1-2 \epsilon) \Delta\right) /(1-2 \epsilon)^{2}}}{2 \epsilon-1},
\end{aligned}
$$

from which one can establish (i)-(iv).

In Figure 1(a), we illustrate the stable, unstable, and saddle regions for the fixed point $(0,0)$. Figures $1(\mathrm{~b})$ and 1 (c) show these three regions for the fixed points $(((r-1) / r),((r-1) / r))$, and $\left(x^{*}, y^{*}\right)$.

In Figure 1(a), the upper curve $((r+1) / 2 r)$ is the flip curve and $((r-1) / 2 r)$ and $r=1$ are fold curves. In Figure 1(b), the two upper dashed curves denote flip and fold curves, respectively, as well as the lines $r=3$ and $r=1$, respectively. In Figure 1(c) we define $h_{1}=((r-1) / 2 r), h_{2}=((r-1) / 2(r-$ $1)), g_{1}=(1 / 2)+(\sqrt{3} / 2) \sqrt{1 / r(r-2)}, \quad g_{2}=(1 / 2)-(\sqrt{3} / 2)$ $\sqrt{1 / r(r-2)}$, and $f_{2}=f_{2}(r)$, as was defined earlier. Here, $g_{1}$ and $g_{2}$ are flip curves, $f_{2}(r)$ is a Neimark-Sacker curve, and $h_{1}$ and $h_{2}$ are the curves bounding the saddle regions. We also note that, for the two symmetric fixed points, we have symmetric regions of stability/instability whose bounding curves exhibit the translation symmetry $\epsilon \mapsto 1-\epsilon$ inherent in the system's defining equations. For the antisymmetric fixed point $\left(x^{*}, y^{*}\right)$, this translation symmetry manifests in the equations for the bounding curves $g_{1}$ and $g_{2}$ but not in the regions bounded by these curves.

\section{Local Bifurcation Analysis}

4.1. Flip Bifurcation. Now, we determine the stability of the nonhyperbolic fixed point $\left(x^{*}, y^{*}\right)$ via center manifold theory. 


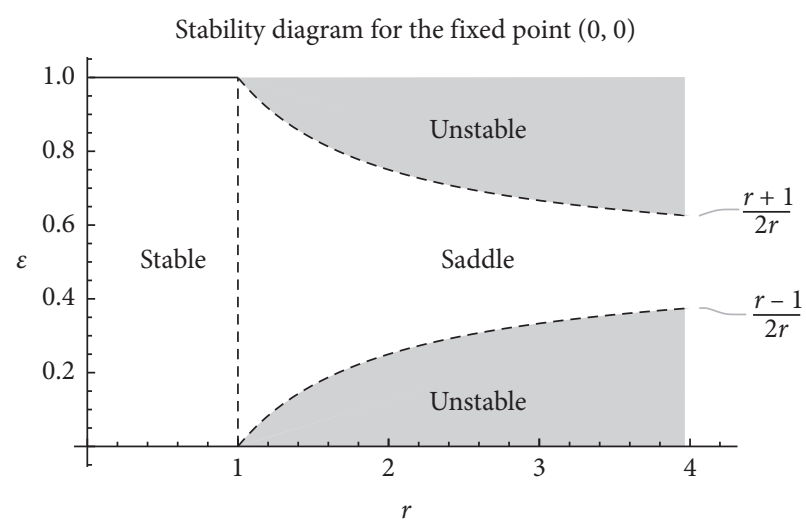

(a)

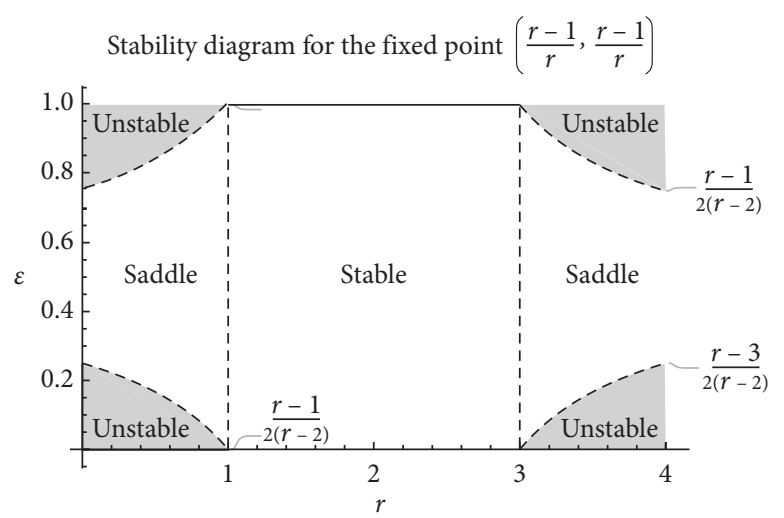

(b)

Stability diagram for the fixed point $(x *, y *)$

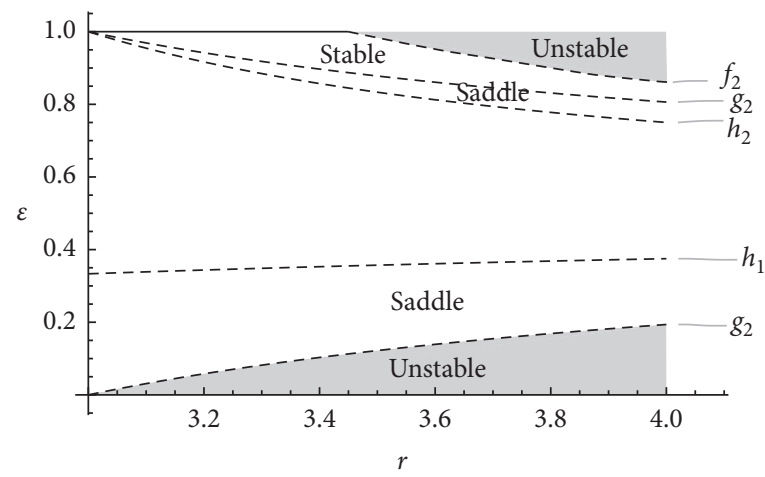

(c)

FIgURE 1: Diagrams of the regions of stability for three of the four fixed points of system $(3)$ in the $(r, \varepsilon)$ plane.

In particular, we demonstrate that system (3) undergoes a flip bifurcation at $\left(x^{*}, y^{*}\right)$, where $\lambda_{1}=-1$ and $\lambda_{2}=((4 \epsilon-$ $3) /(2 \epsilon-1))$ and where we choose $\epsilon$ as our bifurcation parameter and allow it to vary in a small neighborhood of $\left(x^{*}, y^{*}\right)$. Generically, a flip bifurcation is characterized by the loss of stability of a periodic orbit as a parameter crosses a critical value from above or below. The flip bifurcation is supercritical if, locally, there exist stable periodic orbits with double the period for parameter values near the critical value forming a new branch that emerges at this value. If unstable periodic orbits with double the period coalesce with and are destroyed by stable periodic orbits, the flip bifurcation is subcritical. Moreover, a flip bifurcation occurs at an eigenvalue of -1 of the Jacobian of the map.

In order to apply center manifold theory, we assume that our discrete system has the form

$$
\begin{aligned}
& x_{n+1}=A x_{n}+F\left(x_{n}, y_{n}\right), \\
& y_{n+1}=B y_{n}+F\left(x_{n}, y_{n}\right),
\end{aligned}
$$

where all of the eigenvalues of the matrix $A$ (an $n \times n$ matrix) are on the unit circle and the eigenvalues of the matrix $B$ (an $m \times m$ matrix) are within the unit circle, and the Jacobian matrix for the system has the form

$$
\left[\begin{array}{ll}
A & 0 \\
0 & B
\end{array}\right]
$$

We assume without loss of generality that the system has the origin as a fixed point. We use a slight modification of the following version of the center manifold theorem in [16]:

Theorem 2. There exists a $C^{r}$-center manifold for system (25) that can be represented locally as

$$
\begin{aligned}
W_{\mathrm{loc}}^{\mathrm{c}}(0,0)= & \left\{(x, y, \mu) \in \mathbb{R}^{3}|y=h(x, \mu),| x \mid<\delta_{1},\right. \\
& \left.|\mu|<\delta_{2}, h(0,0)=\operatorname{Dh}(0,0),|x|<\epsilon,|\mu|<\delta\right\} .
\end{aligned}
$$

Furthermore, the dynamics of the system restricted to $W_{\text {loc }}^{\text {c }}(0)$ are given locally by the map

$$
x \longmapsto A x+f(x, \mu, h(x, \mu)), \quad \text { for } x \in \mathbb{R} .
$$

In addition, we state the following theorem from [15] which gives criteria for the existence of a flip bifurcation.

Theorem 3. Let $f_{\mu}: \mathbb{R} \longrightarrow \mathbb{R}$ be a one parameter family of mappings such that $f_{\mu_{0}}$ has a fixed point $x_{0}$ with an eigenvalue of value -1. Assume

$$
\begin{array}{r}
\frac{\partial f}{\partial \mu} \frac{\partial^{2} f}{\partial x^{2}}+2 \frac{\partial^{2} f}{\partial x \partial \mu} \neq 0, \quad \text { at }\left(x_{0}, \mu_{0}\right), \\
\frac{1}{2}\left(\frac{\partial^{2} f}{\partial x^{2}}\right)^{2}+\frac{1}{3}\left(\frac{\partial^{3} f}{\partial x^{3}}\right) \neq 0, \quad \text { at }\left(x_{0}, \mu_{0}\right) .
\end{array}
$$


Then, there is a smooth curve of fixed points of $f_{\mu}$ passing through $\left(x_{0}, \mu_{0}\right)$, the stability of which changes at $\left(x_{0}, \mu_{0}\right)$. There is also a smooth curve $\gamma$ passing through $\left(x_{0}, \mu_{0}\right)$ so that $\gamma-\left(x_{0}, \mu_{0}\right)$ is a union of hyperbolic period 2 orbits. The curve $\gamma$ has quadratic tangency with the line $\mathbb{R} \times$ $\left\{\mu_{0}\right\}$ at $\left(x_{0}, \mu_{0}\right)$.

We begin the establishment of a flip bifurcation at $\left(x^{*}, y^{*}\right)$ by first defining the set

$$
H_{F P}=\left\{(r, \epsilon): r \in[3,4), \epsilon=\frac{1}{2}+\frac{\sqrt{3}}{2} \sqrt{\frac{1}{r(r-2)}}\right\} \text {, }
$$

containing the parameters that satisfy the second condition for a hyperbolic point in C (iv) from Theorem 1. Suppose the parameters $\left(r_{s}, \epsilon_{s}\right) \in H_{F P}$ are arbitrarily chosen. Consider the change of variables $u_{n}=x_{n}-x^{*}$ and $v_{n}=y_{n}-y^{*}$. Furthermore, define $\bar{\epsilon}=\epsilon-\epsilon_{s}$ to be a new independent variable, where $\epsilon_{s}=(1 / 2)+(\sqrt{3} / 2)$ $\sqrt{1 / r(r-2)}$. Here, we transform the fixed point $\left(x^{*}, y^{*}\right)$ into $(0,0)$. System (3) now has the form

$$
\left(\begin{array}{c}
u_{n+1} \\
v_{n+1}
\end{array}\right)=\left(\begin{array}{l}
a_{11} u_{n}+a_{12} v_{n}+a_{13} u_{n}^{2}+a_{14} v_{n}^{2}+b^{*} \bar{\epsilon}+b_{13} \bar{\epsilon} u_{n}^{2}-b_{13} \bar{\epsilon} v_{n}^{2} \\
a_{21} u_{n}+a_{22} v_{n}+a_{23} u_{n}^{2}+a_{24} v_{n}^{2}-b^{*} \bar{\epsilon}-b_{13} \bar{\epsilon} u_{n}^{2}+b_{13} \bar{\epsilon} v_{n}^{2}
\end{array}\right),
$$

where

$$
\begin{aligned}
& a_{11}=r_{s}\left(1-\epsilon_{s}\right)\left(1-2 x^{*}\right), \\
& a_{12}=r_{s} \epsilon_{s}\left(1-2 y^{*}\right), \\
& a_{13}=r_{s}\left(\epsilon_{s}-1\right),
\end{aligned}
$$

$$
\begin{aligned}
a_{14}= & -r_{s} \epsilon_{s}, \\
a_{21}= & r_{s} \bar{\epsilon}\left(1-2 x^{*}\right), \\
a_{22}= & r_{s}\left(1-e_{s}\right)\left(1-2 y^{*}\right), \\
b^{*}= & r_{s} \bar{\epsilon}\left(\left(x^{*}\right)^{2}-x^{*}+y^{*}-\left(y^{*}\right)^{2}\right. \\
& \left.-\left(1-2 x^{*}\right) u_{n}+\left(1-2 y^{*}\right) v_{n}\right), \\
b_{13}= & r_{s} .
\end{aligned}
$$

We begin the process of putting the system into the format of the equations in (25) by first defining an invertible matrix:

$$
T=\left(\begin{array}{cc}
-a_{12} & -a_{12} \\
a_{11}+1 & a_{11}-\lambda_{2}
\end{array}\right) .
$$

Determined by the eigenvectors associated with the linearization of the system at $(0,0)$, using the transformation,

$$
\left(\begin{array}{c}
u_{n} \\
v_{n}
\end{array}\right)=T\left(\begin{array}{c}
X_{n} \\
Y_{n}
\end{array}\right)
$$

and letting $\mu=\bar{\epsilon}$, the system now takes the desired form:

$$
\left(\begin{array}{c}
X_{n+1} \\
Y_{n+1}
\end{array}\right)=\left(\begin{array}{cc}
-1 & 0 \\
0 & \lambda_{2}-a_{11}
\end{array}\right)\left(\begin{array}{l}
X_{n} \\
Y_{n}
\end{array}\right)+\left(\begin{array}{c}
F\left(X_{n}, Y_{n}, \mu\right) \\
G\left(X_{n}, Y_{n}, \mu\right)
\end{array}\right),
$$

where

$$
\begin{aligned}
F\left(X_{n}, Y_{n}, \mu\right)= & \frac{b_{2}}{a_{12}\left(1+\lambda_{2}\right)}\left[\left(a_{13} a_{12}^{2}-a_{13} b_{1}\right) X_{n}^{2}+\left(\left(a_{13} a_{12}^{2}-a_{13} b_{2}\right) Y_{n}^{2}+\left(2 a_{13}\left(a_{12}^{2}-b_{1} b_{2}\right) X_{n} Y_{n}+\mu b^{*}\right]\right.\right. \\
& +\frac{b_{2}}{a_{12}\left(1+\lambda_{2}\right)}\left[b_{13} \mu\left(\left[a_{12}^{2}-b_{1}^{2}\right] X_{n}^{2}+\left[a_{12}^{2}-b_{2}^{2}\right] Y_{n}^{2}+2\left[a_{12}^{2}-b_{1} b_{2}\right] X_{n} Y_{n}\right)\right] \\
& +\frac{1}{1+\lambda_{2}}\left[\left(a_{13}\left(b_{1}-a_{12}^{2}\right)\right) X_{n}^{2}+\left(a_{13}\left(b_{2}-a_{12}^{2}\right)\right) Y_{n}^{2}+\left(2 a_{13}\left(b_{1} b_{2}-a_{12}^{2}\right) X_{n} Y_{n}-\mu b^{*}\right]\right. \\
& +\frac{1}{1+\lambda_{2}}\left[-b_{13} \mu\left(\left[a_{12}^{2}-b_{1}^{2}\right] X_{n}^{2}+\left[a_{12}^{2}-b_{2}^{2}\right] Y_{n}^{2}+2\left[a_{12}^{2}-b_{1} b_{2}\right] X_{n} Y_{n}\right)\right], \\
G\left(X_{n}, Y_{n}, \mu\right)= & \frac{-b_{1}}{a_{12}\left(1+\lambda_{2}\right)}\left[a_{13}\left(a_{12}^{2}-b_{1}\right) X_{n}^{2}+a_{13}\left(a_{12}^{2}-b_{2}\right) Y_{n}^{2}+2\left(a_{13}\left(a_{12}^{2}-b_{1} b_{2}\right)\right) X_{n} Y_{n}+\mu b^{*}\right] \\
& \left.\cdot \frac{-b_{1}}{a_{12}\left(1+\lambda_{2}\right)}\left[b_{13} \mu\left[\left(a_{12}^{2}-b_{1}^{2}\right) X_{n}^{2}+\left(a_{12}^{2}-b_{2}^{2}\right) Y_{n}^{2}+2\left(a_{12}^{2}-b_{1} b_{2}\right) X_{n} Y_{n}\right) X_{n} Y_{n}\right]\right] \\
& -\frac{1}{1+\lambda_{2}}\left[a_{13}\left(b_{1}-a_{12}^{2}\right) X_{n}^{2}+a_{13}\left(b_{2}-a_{12}^{2}\right) Y_{n}^{2}+2 a_{13}\left(b_{1} b_{2}-a_{12}^{2}\right) X_{n} Y_{n}-\mu b^{*}\right] \\
& -\frac{1}{1+\lambda_{2}}\left[-b_{13} \mu\left[\left(a_{12}^{2}-b_{1}^{2}\right) X_{n}^{2}+\left(a_{12}^{2}-b_{2}^{2}\right) Y_{n}^{2}+2\left(a_{12}^{2}-b_{1} b_{2}\right) X_{n} Y_{n}\right]\right],
\end{aligned}
$$


where $b_{1}=a_{11}+1$ and $b_{2}=a_{11}-\lambda_{2}$. By applying the center manifold theorem, we see that there exists a center manifold for system (3) defined as

$$
\begin{aligned}
W_{\mathrm{loc}}^{\mathrm{c}}(0,0)= & \left\{(x, y, \mu) \in \mathbb{R}^{\|=}|y=h(x, \mu),| x \mid<\delta_{1},\right. \\
& \left.|\mu|<\delta_{2}, h(0,0)=\operatorname{Dh}(0,0),|x|<\epsilon,|\mu|<\delta\right\},
\end{aligned}
$$

for sufficiently small $\epsilon$ and $\delta$. To actually find the center manifold as the graph of $y=h(x, \mu)$, we consider a power series representation for this map:

$$
y=h(x, \mu)=A_{0} X^{2}+A_{1} X \mu+A_{2} \mu^{2}+O\left((|X|+|\mu|)^{3}\right),
$$

which we then substitute into (25). Hence, the center manifold must satisfy the equation

$$
h(-x+F(x, h(x, \mu), \mu), \mu)=\lambda_{2} h(x, \mu)+G(x, h(x, \mu), \mu) .
$$

Write $F$ in the form

$$
\begin{aligned}
F(X, Y, \mu)= & \left(f_{1}-g_{1}\right)\left[e_{1} X^{2}+e_{2} Y^{2}+e_{3} X Y+\mu e_{4}\right. \\
& \left.+\mu e_{8}\left(e_{5} X^{2}+e_{6} Y^{2}+e_{7} X Y\right)\right],
\end{aligned}
$$

and $G$ in the form

$$
\begin{aligned}
G(X, Y, \mu)= & \left(f_{2}-g_{2}\right)\left[e_{1} X^{2}+e_{2} Y^{2}+e_{3} X Y\right. \\
& \left.+\mu e_{4}+\mu e_{8}\left(e_{5} X^{2}+e_{6} Y^{2}+e_{7} X Y\right)\right],
\end{aligned}
$$

where

$$
\begin{aligned}
& e_{1}=a_{13}\left(a_{12}^{2}-b_{1}\right), \\
& e_{2}=a_{13}\left(a_{12}^{2}-b_{2}\right), \\
& e_{3}=2 a_{13}\left(a_{12}^{2}-b_{1} b_{2}\right), \\
& e_{4}=b^{*}, \\
& e_{5}=a_{12}^{2}-b_{1}^{2}, \\
& e_{6}=a_{12}^{2}-b_{2}^{2}, \\
& e_{7}=2\left(a_{12}^{2}-b_{1} b_{2}\right), \\
& e_{8}=b_{13}, \\
& f_{1}=\frac{b_{2}}{a_{12}\left(1+\lambda_{2}\right)}, \\
& g_{1}=\frac{1}{1+\lambda_{2}}, \\
& g_{2}=-g_{1}, \\
& f_{2}=\frac{-b_{1}}{a_{12}\left(1+\lambda_{2}\right)} .
\end{aligned}
$$

By substituting the equations for $F, G$, and $h$ into the center manifold equation (39) and equating the coefficients of like terms on either side of the equation, we determine the coefficients $A_{0}, A_{1}$, and $A_{2}$ :

$$
\begin{aligned}
& A_{0}=\frac{\left(f_{2}-g_{2}\right) e_{1}}{1-\lambda_{2}}, \\
& A_{1}=\frac{2 A_{0}\left(g_{1}-f_{1}\right) \mu e_{4}}{1+\lambda_{2}}, \\
& A_{2}=\frac{\left(f_{1}-g_{1}\right)\left[A_{0} e_{4}^{2}+A_{1} e_{4}\right]}{\lambda_{2}-1} .
\end{aligned}
$$

The restriction of our map to the center manifold is defined as the map

$$
\begin{aligned}
K(X, \mu):= & -X+\left(f_{1}-g_{1}\right)\left[\left(e_{1}+\mu e_{8} e_{5}\right) X^{2}\right. \\
& \left.+\left(e_{3}+\mu e_{8} e_{7}\right)\left(X^{3}+A_{1} X^{2} \mu+A_{2} \mu^{2} X\right)\right] \\
& +\left(f_{1}-g_{1}\right)\left[( e _ { 2 } + \mu e _ { 8 } e _ { 6 } ) \left(A_{0}^{2} X^{4}+A_{1}^{2} X^{2} \mu^{2}+A_{2}^{2} \mu^{4}\right.\right. \\
& \left.+2 A_{0} A_{2} X^{2} \mu^{2}+2 A_{0} X^{3} A_{1} \mu+2 A_{1} A_{2} \mu^{4} X\right] .
\end{aligned}
$$

Straightforward but detailed calculations show that

$$
\begin{aligned}
\alpha_{1} & =\frac{\partial K}{\partial \mu} \frac{\partial^{2} K}{\partial X^{2}}+\frac{2 \partial^{2} K}{\partial X \partial \mu}=\left.2 e_{1} e_{4}\left(f_{1}-g_{1}\right)^{2}\right|_{(0,0)} \neq 0, \\
\alpha_{2} & =\frac{1}{2}\left(\frac{\partial^{2} K}{\partial X^{2}}\right)^{2}+\frac{1}{3}\left(\frac{\partial^{3} K}{\partial X^{3}}\right) \\
& =\left.2\left(f_{1}-g_{1}\right)\left[\left(f_{1}-g_{1}\right) e_{1}^{2}+A_{0} e_{3}\right]\right|_{(0,0)} \neq 0 .
\end{aligned}
$$

By Theorems 2 and 3 above, the following result is now established:

Theorem 4. If $\alpha_{1}, \alpha_{2} \neq 0$, then the map undergoes a flip bifurcation at the fixed point $\left(x^{*}, y^{*}\right)$ when the parameter $\epsilon$ varies in a small neighborhood of $\epsilon_{s}$. Moreover, if $\alpha_{2}>0$ (respectively, $\alpha_{2}<0$ ), the period 2 orbits that bifurcate from $\left(x^{*}, y^{*}\right)$ are stable (unstable).

4.2. Neimark-Sacker Bifurcation. A Neimark-Sacker bifurcation is characterized by a stable fixed point becoming unstable at a certain critical value of the bifurcation parameter of the system in which an attracting closed invariant curve manifests or a repelling closed invariant curve emerges as the values of the parameter cross this critical value. In the former case, we say the bifurcation is a supercritical Neimark-Sacker bifurcation; in the latter case, a subcritical Neimark-Sacker bifurcation. In either case, such a bifurcation is associated with discrete systems whose eigenvalues are complex conjugates of modulus ones.

Here, we state a slight modification of a theorem from [16] (Chapter 5), which outlines the criteria for the emergence of such a bifurcation. 
Theorem 5 (Neimark-Sacker). Consider the family of $C^{r}$ maps $(r \geq 5), F_{\mu}: \mathbb{R}^{\|=} \times \mathbb{R} \longrightarrow \mathbb{R}^{\|=}$such that the following conditions hold:

(1) $F_{\mu}(0)=0$, i.e., the origin is a fixed point of $F_{\mu}$.

(2) $D F_{\mu}(0)$ has two complex conjugate eigenvalues $\lambda_{1,2}(\mu)=r(\mu) e^{ \pm i \theta(\mu)}$, where $r(0)=1, r^{\prime}(0) \neq 0$, $\theta(0)=\theta_{0}$.

(3) $e^{i k \theta_{0}} \neq 1$ for $k=$

$1,2,3,4$ (absence of strong resonances condition).

If, in addition, $a \neq 0$, where

$$
a=-\operatorname{Re}\left[\frac{(1-2 \lambda) \bar{\lambda}^{2} \zeta_{11} \zeta_{20}}{1-\lambda}\right]-\frac{1}{2}\left|\zeta_{11}\right|^{2}-\left|\zeta_{02}\right|^{2}+\operatorname{Re}\left(\bar{\lambda} \zeta_{21}\right)
$$

( $a$ is called the first Lyapunov coefficient),

then, for sufficiently small $\mu$ and $F_{\mu}$, there exists a unique invariant closed curve enclosing that bifurcates from the origin as a passes through 0 . If $a<0$, we have a supercritical Neimark-Sacker bifurcation. If $a>0$, we have a subcritical Neimark-Sacker bifurcation. The complex conjugate eigenvalues of our system are given by the following formulas:

$$
\lambda_{i}=\frac{\epsilon-1 \pm i(2 \epsilon-1) \sqrt{\left(-\epsilon^{2}+(2 \epsilon-1) \Delta\right) /(1-2 \epsilon)^{2}}}{2 \epsilon-1},
$$

for $i=1,2$.

$$
\left|\lambda_{i}\right|=1 \text {, for } i=1,2 \text { if and only if, } \sqrt{\frac{\Delta-1}{2 \epsilon-1}}=1 \text { or } \Delta=2 \epsilon \text {. }
$$

Thus, the range of parameters, for which the eigenvalues associated with the fixed point $\left(x^{*}, y^{*}\right)$ are complex conjugates and have magnitude 1 , can be described by the set:

$$
\begin{aligned}
H_{N S} & =\left\{(r, \epsilon): \epsilon^{2}+(1-2 \epsilon) \Delta<0, \Delta=2 \epsilon\right\} \\
& \equiv\left\{(r, \epsilon): r \in[1+\sqrt{6}, 4), \epsilon=f_{2}(r)\right\} .
\end{aligned}
$$

We now show that a Neimark-Sacker bifurcation occurs at $\left(x^{*}, y^{*}\right)$ for arbitrary parameters $\left(e_{h}, r_{h}\right) \in H_{N S}$, taking $\epsilon$ as our bifurcation parameter and allowing it to vary in a small neighborhood of $e_{h}$. So, we consider a small perturbation of the parameter $\epsilon$ as follows: $\overline{\bar{\epsilon}}=\epsilon-\epsilon_{h}$ and transform the fixed point $\left(x^{*}, y^{*}\right)$ to the origin $(0,0)$, as before, to produce the system (where we are essentially replacing $e_{s}$ by $e_{h}$ in an earlier statement of our system) with coefficients that were defined in Section 3:

$$
\left(\begin{array}{l}
u_{n+1} \\
v_{n+1}
\end{array}\right)=\left(\begin{array}{l}
a_{11} u_{n}+a_{12} v_{n}+a_{13} u_{n}^{2}+a_{14} v_{n}^{2}+b^{*} \overline{\bar{\epsilon}}+b_{13} \overline{\bar{\epsilon}} u_{n}^{2}-b_{13} \overline{\bar{\epsilon}} v_{n}^{2} \\
a_{21} u_{n}+a_{22} v_{n}+a_{23} u_{n}^{2}+a_{24} v_{n}^{2}-b^{*} \overline{\bar{\epsilon}}-b_{13} \overline{\bar{\epsilon}} u_{n}^{2}+b_{13} \overline{\bar{\epsilon}} v_{n}^{2}
\end{array}\right) \text {. }
$$

Now, the characteristic equation at $\left(u_{n}, v_{n}\right)=(0,0)$ is as follows:

$$
\begin{aligned}
\lambda^{2} & -\lambda\left(r_{h}\left(1-\epsilon_{h}-\overline{\bar{\epsilon}}\right)\left(1-2 x^{*}\right)+r_{h}\left(\epsilon_{h}+\overline{\bar{\epsilon}}\right)\left(1-2 y^{*}\right)\right) \\
& +r_{h}^{2}\left(1-2\left(\epsilon_{h}-\overline{\bar{\epsilon}}\right)\right)\left(1-2 x^{*}\right)\left(1-2 y^{*}\right),
\end{aligned}
$$

where

A simple calculation shows that

$$
\lambda_{1,2}=\frac{\left(\epsilon_{h}+\overline{\bar{\epsilon}}\right)-1 \pm i\left(2\left(\epsilon_{h}+\overline{\bar{\epsilon}}\right)-1\right) \sqrt{\left(-\left(\epsilon_{h}+\overline{\bar{\epsilon}}\right)^{2}+\left(2\left(\epsilon_{h}+\overline{\bar{\epsilon}}\right)-1\right) \Delta\right) /\left(1-2\left(\epsilon_{h}+\overline{\bar{\epsilon}}\right)\right)^{2}}}{2\left(\epsilon_{h}+\overline{\bar{\epsilon}}\right)-1} .
$$

A straightforward calculation shows that

$$
\begin{aligned}
& \frac{\mathrm{d}}{\mathrm{d} \overline{\bar{\epsilon}}}\left(\left|\lambda_{1,2}\right|\right)=\left.\frac{\mathrm{d}}{\mathrm{d} \overline{\bar{\epsilon}}}\left(\sqrt{\frac{\widetilde{\Delta}-1}{\left(2\left(\overline{\bar{\epsilon}}+\epsilon_{h}\right)-1\right)}}\right)\right|_{\overline{\bar{\epsilon}}}=0 \\
& =\frac{2}{\left(1-2 \epsilon_{h}\right)^{2}}+\left(r_{h}\right)^{2}-2 r_{h}>0, \\
& \quad \text { for }\left(r_{h}, \epsilon_{h}\right) \in H_{N S},
\end{aligned}
$$

where

$$
\widetilde{\Delta}=\left(1-4\left(\overline{\bar{\epsilon}}+\epsilon_{h}\right)\right)(r-1)^{2}+4\left(\overline{\bar{\epsilon}}+\epsilon_{h}^{2}\right) r_{h}\left(r_{h}-2\right) 2\left(\overline{\bar{\epsilon}}+\epsilon_{h}\right) \text {. }
$$

Now, we state conditions for the absence of strong resonances, i.e., $\lambda_{1,2}^{m}\left(\epsilon_{h}\right) \neq 1, m=1,2,3,4$ for $\overline{\bar{\epsilon}}=0$. Here, we note that the condition that the eigenvalues are a pair of complex conjugates leads to the following condition deducible from equation (34), using $\Delta=2 \epsilon$.
We can write

$$
\lambda_{1,2}=\frac{\epsilon-1 \pm i(2 \epsilon-1) \sqrt{\left(3 \epsilon^{2}-2 \epsilon\right) /(1-2 \epsilon)^{2}}}{2 \epsilon-1} .
$$

An examination of the condition $\lambda^{m}\left(e_{h}\right) \neq 1$ for $m=1,2,3,4$, leads to the constraints $\epsilon \neq 0,(2 / 3),(3 / 4), 1$. For $r \in[1+\sqrt{6}, 4)$, these $\epsilon$ constraints, for $\epsilon \in H_{N S}$, are equivalent to $r \neq 1+\sqrt{6}$ which we now require. Now, we study the normal form of our system when $\overline{\bar{\epsilon}}=0$ by first computing the following Taylor expansion at $\left(u_{n}, v_{n}\right)=(0,0)$ :

$$
\left(\begin{array}{l}
u_{n+1} \\
v_{n+1}
\end{array}\right)=\left(\begin{array}{l}
a_{11} u_{n}+a_{12} v_{n}+a_{13} u_{n}^{2}+a_{14} v_{n}^{2} \\
a_{21} u_{n}+a_{22} v_{n}+a_{14} u_{n}^{2}+a_{13} v_{n}^{2}
\end{array}\right),
$$

where the coefficients $a_{11}, a_{21}, a_{12}, a_{13}, a_{14}, a_{22}$ were defined earlier. Next, we define $A_{1}=((\epsilon-1) /(2 \epsilon-1))$ and $A_{2}=\sqrt{\left(3 \epsilon^{2}-2 \epsilon\right) /(1-2 \epsilon)^{2}}$; these coefficients represent the 
real and imaginary parts of $\lambda_{1,2}$. Upon finding the eigenvectors associated with these eigenvalues, we construct the following invertible matrix:

$$
T=\left(\begin{array}{cc}
-a_{12} & 0 \\
a_{11}-A_{1} & A_{2}
\end{array}\right) .
$$

Using the transformation,

$$
\left(\begin{array}{c}
u_{n} \\
v_{n}
\end{array}\right)=T\left(\begin{array}{l}
X_{n} \\
Y_{n}
\end{array}\right)
$$

$$
\begin{aligned}
& A_{3}=\frac{A_{1}^{2}-A_{1}\left(a_{11}+a_{22}\right)+a_{11} a_{22}-a_{21} a_{12}}{A_{2}}, \\
& A_{4}=a_{11}+a_{22}-A_{1}, \\
& c_{21}=\frac{A_{1}^{2} a_{13}-2 A_{1} a_{11} a_{13}+\left(a_{11}\right)^{2} a_{13}-A_{1} a_{12} a_{23}+a_{11} a_{12} a_{23}+a_{12}^{2} a_{23}}{A_{2}}+\frac{a_{11}^{3} a_{13}+3 A_{1}^{2} a_{11} a_{13}-A_{1}^{3} a_{13}-3 A_{1} a_{11}^{2} a_{13},}{a_{12} A_{2}}, \\
& c_{22}=2 a_{11} a_{13}-2 A_{1} a_{13}+\frac{2 A_{1}^{2} a_{13}-4 A_{1} a_{11} a_{13}+2 a_{11}^{2} a_{13}}{a_{12}}, \\
& c_{23}=A_{2} a_{13}+\frac{-A_{1} A_{2} a_{13}+a_{11} A_{2} a_{13}}{a_{12}}, \\
& c_{11}=\frac{2 A_{1} a_{11} a_{23}-A_{1}^{2} a_{13}-a_{11}^{2} a_{23}}{a_{12}}, \\
& c_{12}=\frac{2 A_{1} A_{2} a_{23}-2 a_{11} A_{2} a_{23}}{a_{12}}, \\
& c_{13}=\frac{A_{2}^{2} a_{23}}{a_{12}} .
\end{aligned}
$$

In addition, we have

$$
\begin{aligned}
\left.F_{x_{n} x_{n}}\right|_{(0,0)} & =2 c_{11}, \\
\left.F_{x_{n} y_{n}}\right|_{(0,0)} & =c_{12} \\
\left.F_{y_{n} y_{n}}\right|_{(0,0)} & =2 c_{13} \\
\left.F_{x_{n} x_{n} x_{n}}\right|_{(0,0)} & =\left.F_{x_{n} x_{n} y_{n}}\right|_{(0,0)}=F_{\left.x_{n} y_{n} y_{n}\right|_{(0,0)}}=F_{\left.y_{n} y_{n} y_{n}\right|_{(0,0)}}=0 \\
\left.G_{x_{n} x_{n}}\right|_{(0,0)} & =2 c_{21}, \\
\left.G_{x_{n} y_{n}}\right|_{(0,0)} & =c_{22} \\
\left.G_{y_{n} y_{n}}\right|_{(0,0)} & =2 c_{23}, \\
\left.G_{x_{n} x_{n} x_{n}}\right|_{(0,0)} & =\left.G_{x_{n} x_{n} y_{n}}\right|_{(0,0)}=\left.G_{x_{n} y_{n} y_{n}}\right|_{(0,0)}=\left.G_{y_{n} y_{n} y_{n}}\right|_{(0,0)}=0 .
\end{aligned}
$$

For $\lambda, \bar{\lambda}=e^{ \pm i \theta}$, we must now show that $a \neq 0$, where
The system can be rendered in the form

$$
\begin{aligned}
& X_{n+1}=A_{1} X_{n}-A_{2} Y_{n}+F\left(X_{n}, Y_{n}\right), \\
& Y_{n+1}=A_{3} X_{n}+A_{4} Y_{n}+G\left(X_{n}, Y_{n}\right),
\end{aligned}
$$

where

$$
\begin{aligned}
& F\left(X_{n}, Y_{n}\right)=c_{11} X_{n}^{2}+c_{12} X_{n} Y_{n}+c_{13} Y_{n}^{2}, \\
& G\left(X_{n}, Y_{n}\right)=c_{21} X_{n}^{2}+c_{22} X_{n} Y_{n}+c_{23} Y_{n}^{2} .
\end{aligned}
$$

Here, the coefficients are defined as 
We summarize our work now as a theorem indicating that a Neimark-Sacker bifurcation occurs at $\left(x^{*}, y^{*}\right)$ and elucidate the nature of the resulting bifurcation curve:

Theorem 6. If $r \neq 1+\sqrt{6}$ and $a \neq 0$ then the map undergoes $a$ Neimark-Sacker bifurcation at the fixed point $\left(x^{*}, y^{*}\right)$, when the parameter $\epsilon$ varies in a small neighborhood of $\epsilon_{h}$. Moreover, if $a<0$ (respectively, $a>0$ ), then an attracting (respectively, repelling) invariant closed curve bifurcates from the fixed point for $\epsilon>\epsilon_{h}$ (respectively, $\epsilon<\epsilon_{h}$ ).

\section{Numerical Results}

In this section, we use mathematics to numerically verify and illustrate the conclusions of Theorems 1, 4, and 6, with respect to the fixed point $\left(x^{*}, y^{*}\right)$.

Using the flip equation $\epsilon=(1 / 2)+(\sqrt{3} / 2) \sqrt{1 / r(r-2)}$ for $r=3.6$, we have $\epsilon=0.860844$ and $\left(x^{*}, y^{*}\right)=$ $(0.548868,0.836032)$ and $\alpha_{2}=-15.6546$. Since the corresponding value $\alpha_{2}<0$, the period-two orbits that bifurcate from $\left(x^{*}, y^{*}\right)$ are unstable and they are succeeded by a stable period-one orbit. In Figure 2, we observe the emergence of the period-one orbit at the bifurcation point. The flip bifurcation occurs at $\epsilon=0.860844$. Here, we include a vertical line at $\epsilon=0.139156$ to show at least numerically that there is another flip bifurcation for $\epsilon=(1 / 2)+(\sqrt{3} / 2) \sqrt{1 / r(r-2)}$. This figure is very similar to the experimental and numerical bifurcation plots contained in [10]. Figure 3 shows that the unstable flip occurs in the chaotic region, that subsequently we get a stable one-cycle thereafter, and that, for even larger $\epsilon$-values, this one-cycle becomes unstable again.

In Figures 4-6, we show further numerical evidence of a flip bifurcation at several other values of $r$. Next, we consider $r=3.1$ which corresponds to $\epsilon=0.968979$. Here, the corresponding fixed point is $(0.611386,0.732523)$ and the value of $\alpha_{2}=-0.225324$. The bifurcation diagram in Figure 4 shows the onset of flip bifurcations at the two marked off vertical lines $\epsilon=0.031021$ and $\epsilon=0.968979$.

Figure 5 gives a sequence of time series plots revealing a stable symmetric two-cycle before the critical value of $\epsilon$ is reached and a weak two-cycle at the critical value of $\epsilon$. In the last plot, we see the emergence of a one-cycle for a value of $\epsilon$ nearby but larger than our critical value. Here, the chosen values of $\epsilon$ are $0.95,0.968979$, and 0.988979 , respectively.

For contrast, we consider a fairly high value of $r=3.83$, deep into the chaotic regime of the system. Here, $\epsilon=0.827119$ and the initial conditions are $(0.24,0.7)$. The fixed point is $(0.533607,0.865478)$ and $\alpha_{2}=-38.6552$ (the corresponding lower value of $\epsilon$, where a flip may occur is $\epsilon=0.172881)$. The accompanying sequence of time series plots shows a chaotic cycle colliding with a two-cycle at our critical value and the birth of a one-cycle for a value of $\epsilon>0.827119$ close to our critical value. Additional time series plots (not included here) in fact show a pattern of intermittency periods of stability and instability of a symmetric and antisymmetric two-cycles colliding before the one-cycle is reached. In the panel, the chosen values of $\epsilon$ are 0.807119 , $0.827119,0.84$, and 0.867 , respectively.

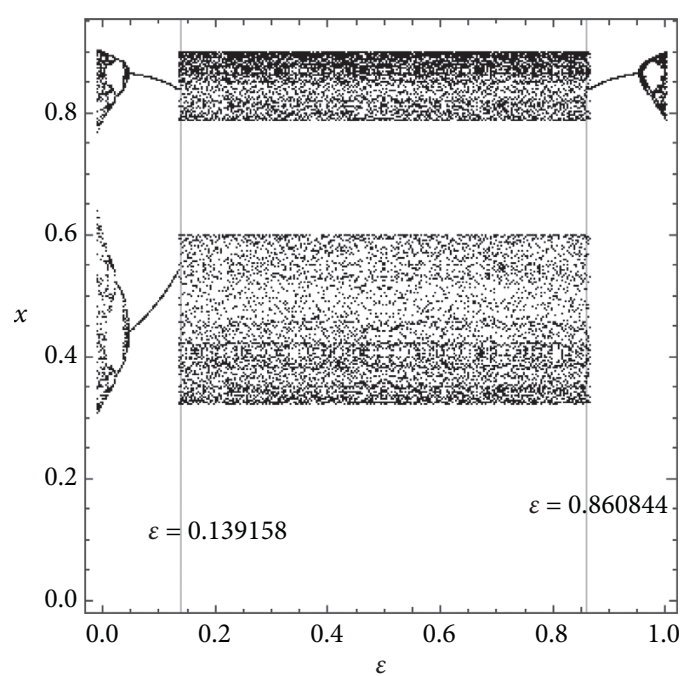

FIGURE 2: Bifurcation diagram for $r=3.6$ and $\varepsilon=0.860844$, initial conditions $(0.18,0.61)$.

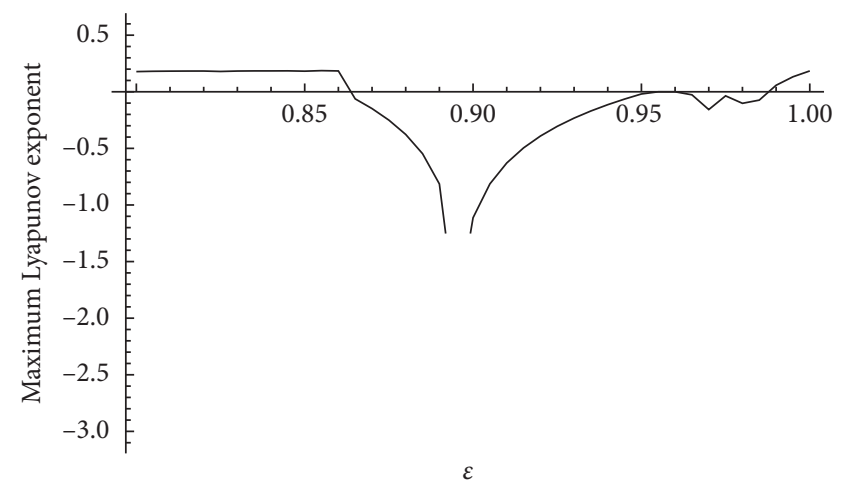

Figure 3: Maximum Lyapunov exponent plot for $r=3.6$.

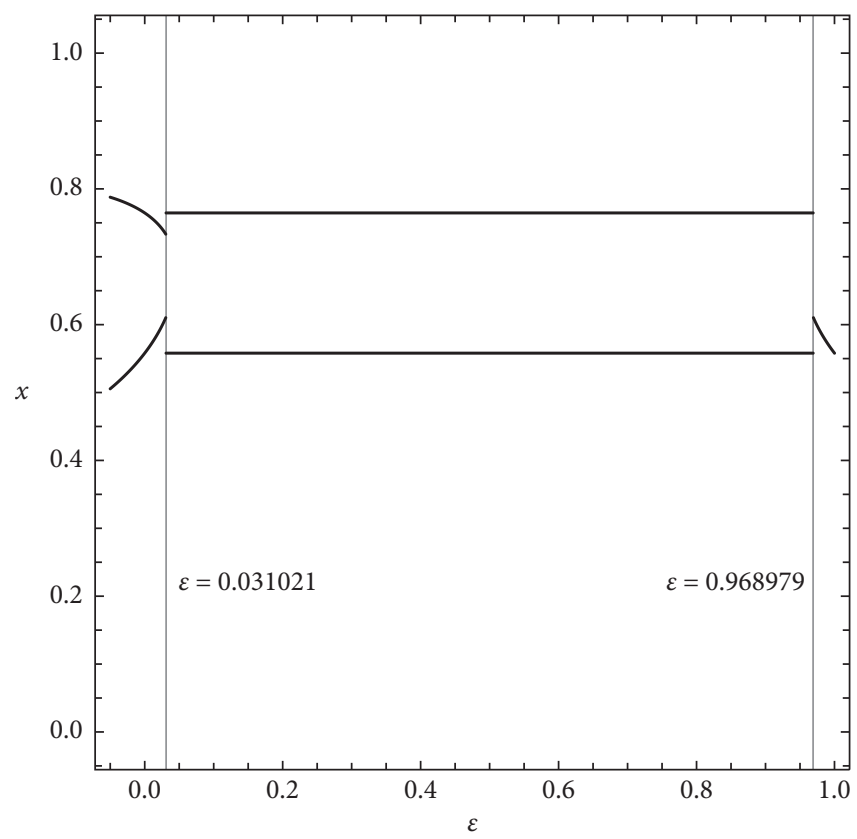

FIGURE 4: Bifurcation diagram for $r=3.1$ and $\varepsilon=0.968979$, with initial conditions $(0.09,0.18)$. 


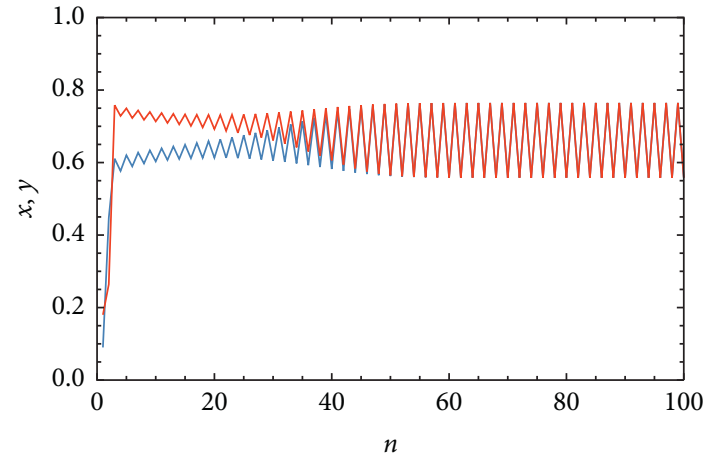

(a)

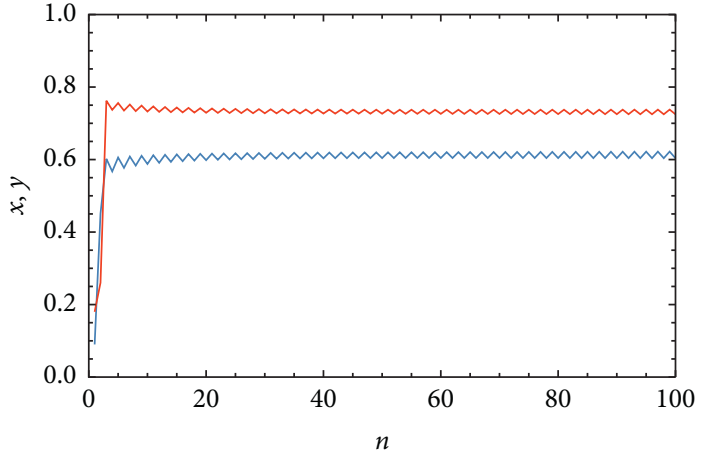

(b)

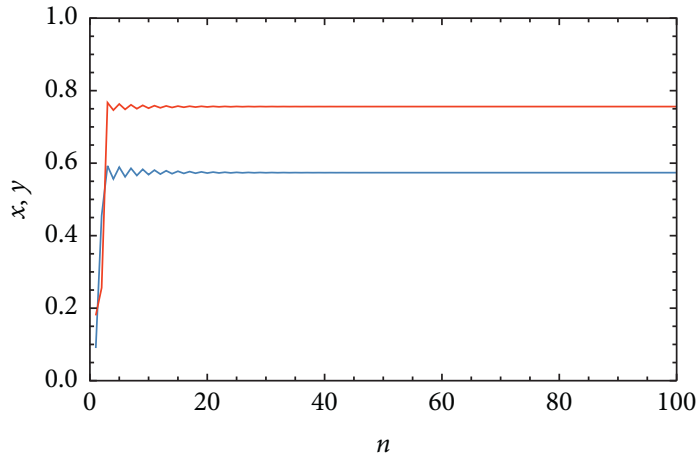

(c)

Figure 5: Time series plots for $r=3.1$ and $\varepsilon=0.95,0.968979,0.988979$, respectively.

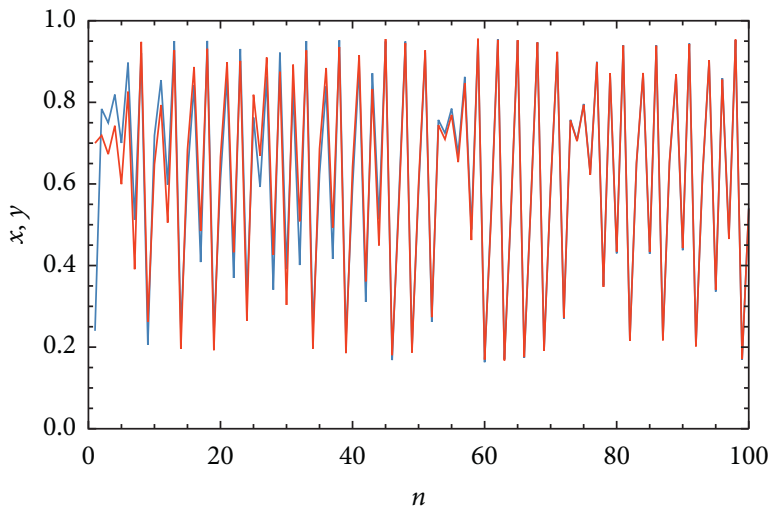

(a)

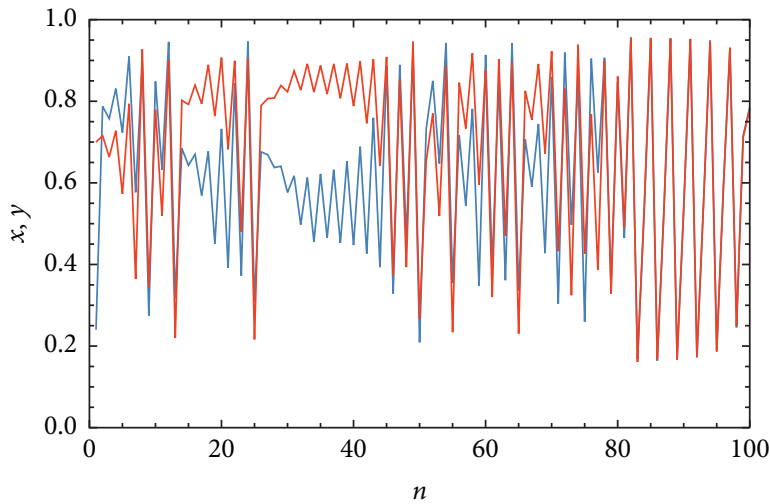

(c)

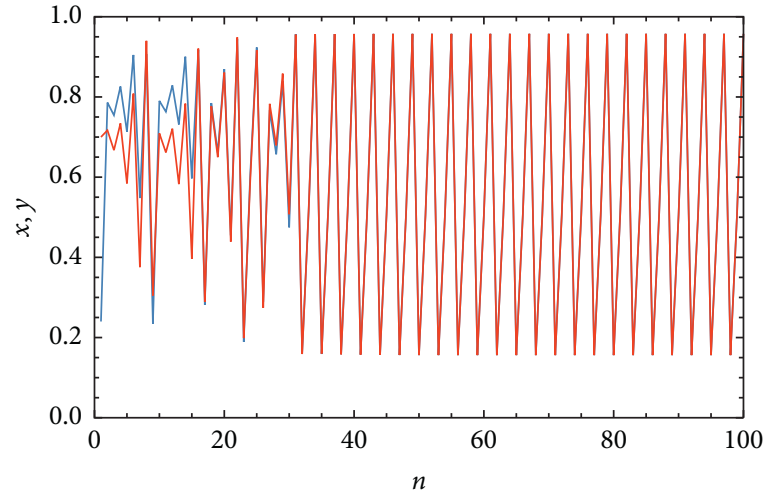

(b)

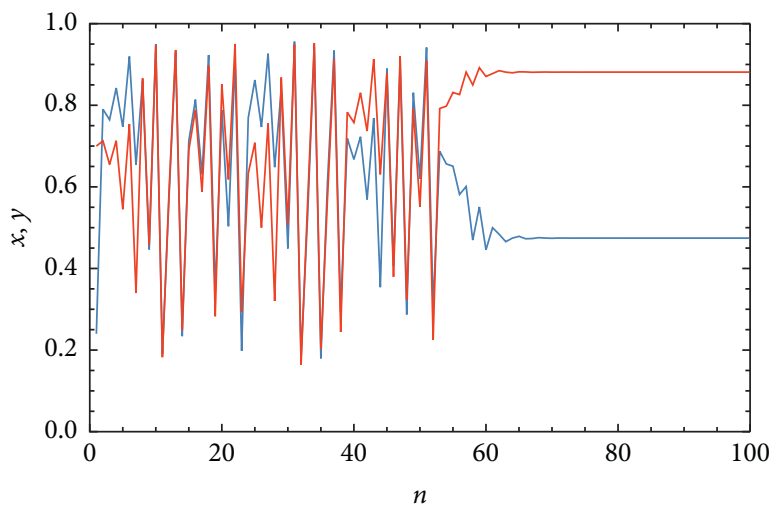

(d)

FIGURE 6: Time series plots for $r=3.83$ and $\varepsilon=0.807119,0.827119,0.84,0.867$, respectively. 


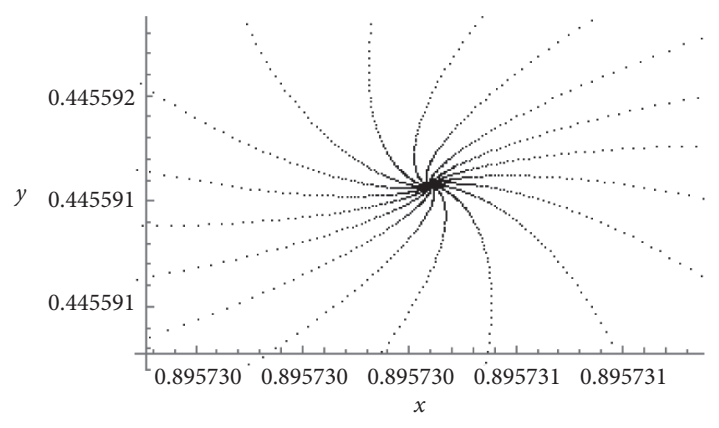

(a)

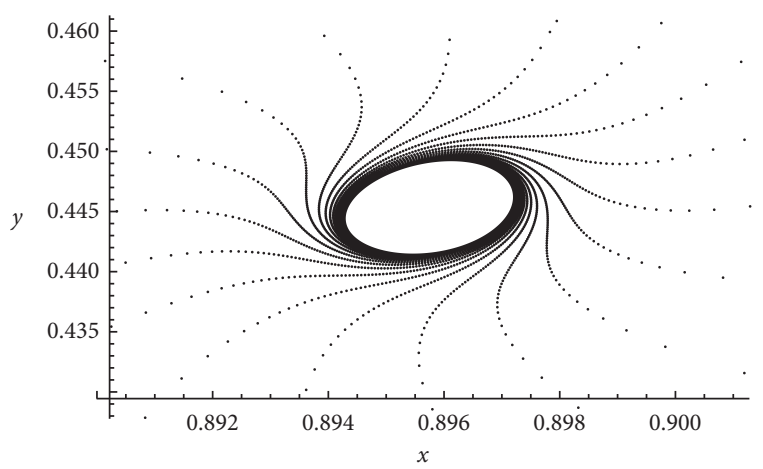

(c)

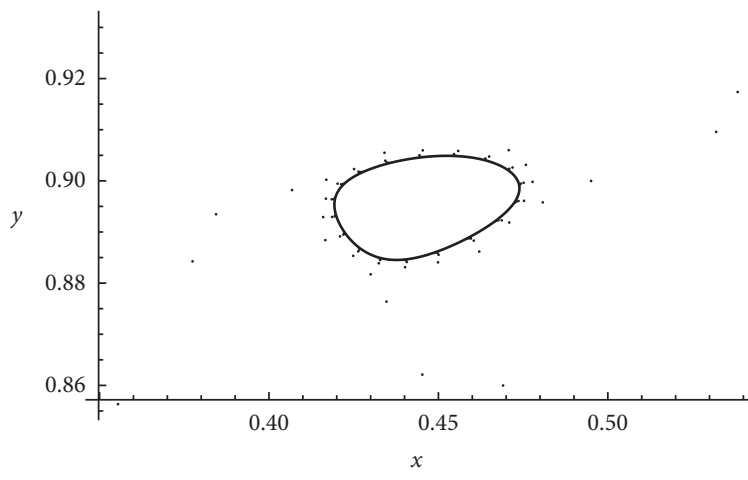

(e)

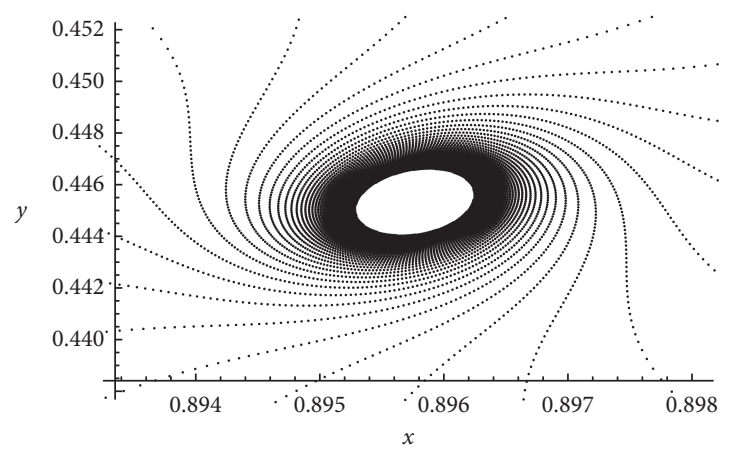

(b)

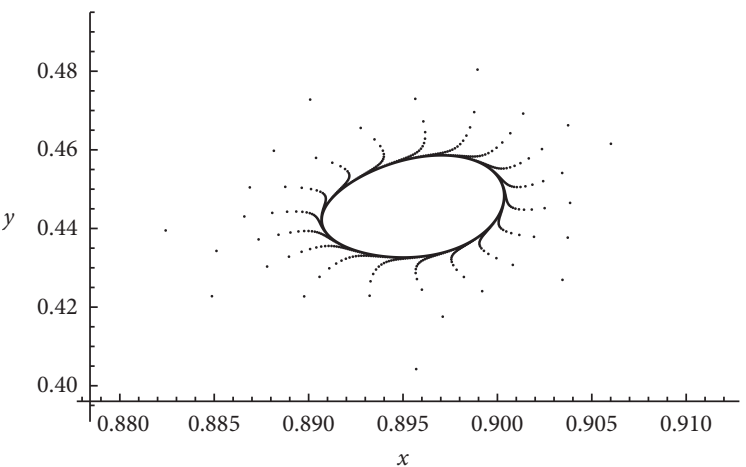

(d)

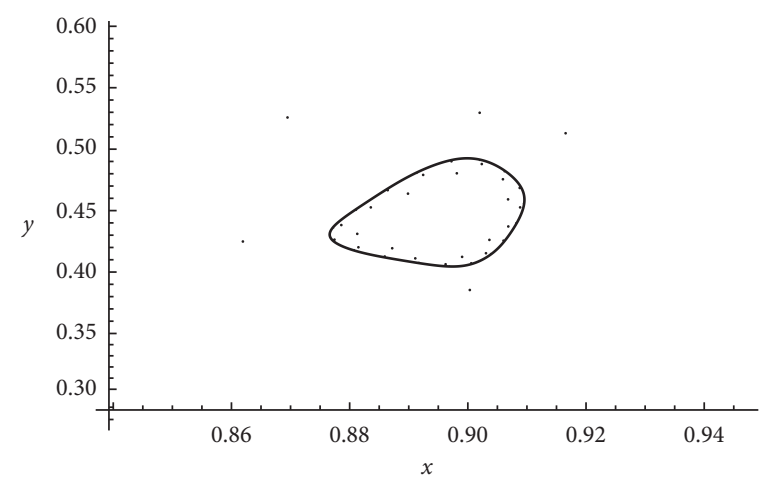

(f)

FIgURE 7: Formation of a Neimark-Sacker bifurcation.

A similar picture emerges also at $r=3.5$ and $r=3.6$. In these cases, below $\epsilon_{c}$, the nonsymmetric two-cycle evolves into the symmetric four-cycle (or quasi-four-cycle for $r=3.6)$ with time, whereas above $\epsilon_{c}$, it evolves into the nonsymmetric one-cycle.

Using the relation $\epsilon=f_{2}(r)$ and substituting 3.94 for $r$, we get that $\epsilon=0.872059$ and the fixed point $\left(x^{*}, y^{*}\right)=(0.445316,0.895769)$. Figures 7 and 8 below show the formation of a Neimark-Sacker bifurcation and chaotic regions in the phase plane for the initial conditions $(0.1,0.3)$.

In Figure $7(\mathrm{a})$, where $\epsilon=0.8718<0.872059$, the fixed point is stable. Figure 7(b) illustrates the loss of stability of the fixed point at $\epsilon=0.872059$.

In Figures $7(\mathrm{c})-7(\mathrm{f}), \quad \epsilon=0.8721,0.8725,0.874,0.877$, respectively. Mhiri et al. [10] found a very similar progression of phase portraits, as the coupling increased.
Here, we see that, for increasing $\epsilon>0.872059$ relatively close to $\epsilon=0.872059$, the gradual development of a closed invariant curve, in other words, a supercritical Neimark-Sacker bifurcation occurs. In addition, a detailed computation of $a$ yields a negative value. Furthermore, Figures $8(a)-8(d)$ (where $\epsilon=0.885,0.888,0.89,0.92$ ) show the transition to a chaotic state with the appearance of 11 coexisting chaotic attractors in Figure 8(b) and a chaotic attracting set in Figures 8(c) and 8(d), for values of $\epsilon$ further away from 0.872059 .

The vertical line in the accompanying bifurcation diagram shows the birth of Neimark-Sacker bifurcation. A plot of the maximum Lyapunov exponent for $r=3.94$ for $\epsilon$ in the range $[0.8,1]$ is also included (Figure 9).

Negative exponents indicate stable regions within the otherwise chaotic regime and positive exponents of the chaotic regions. 


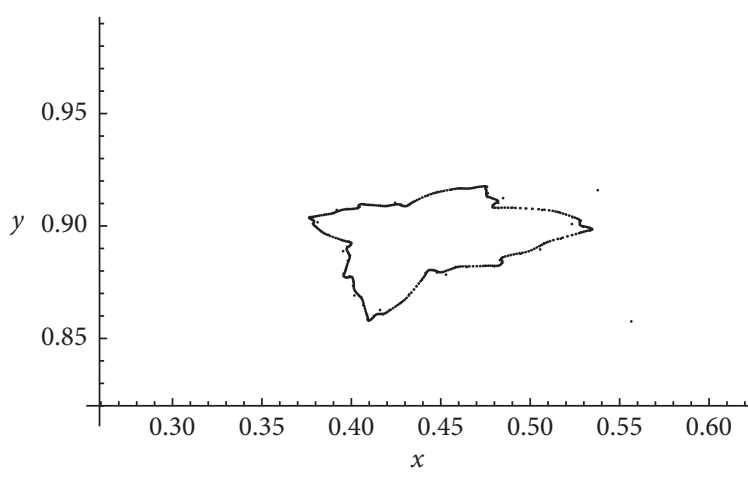

(a)

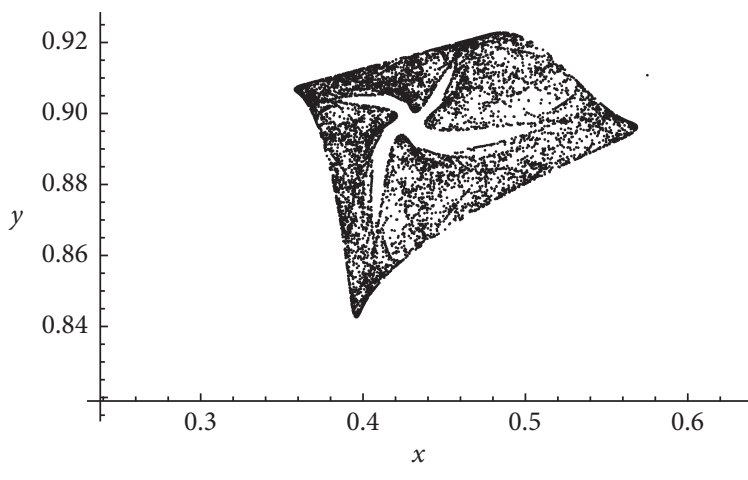

(c)

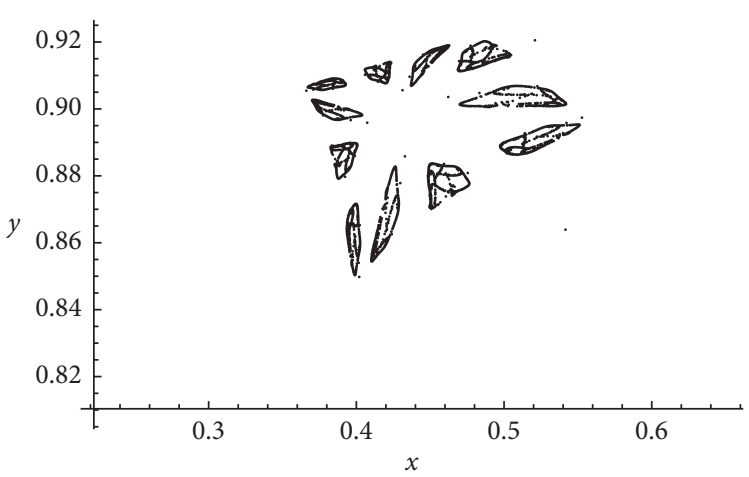

(b)

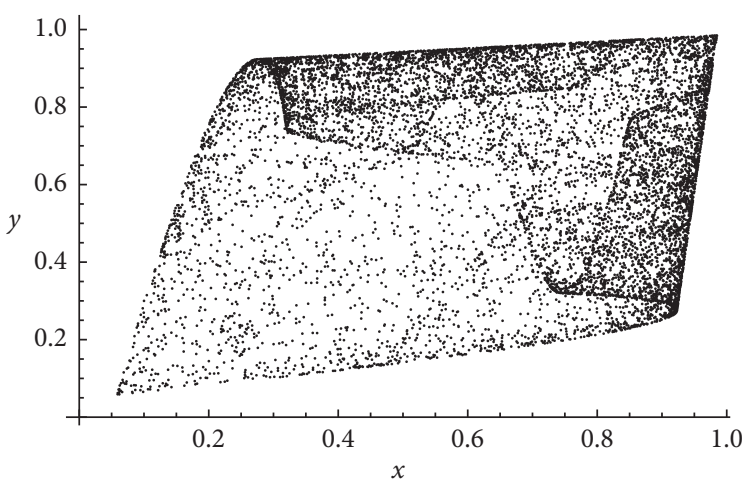

(d)

Figure 8: Emergence of chaos.

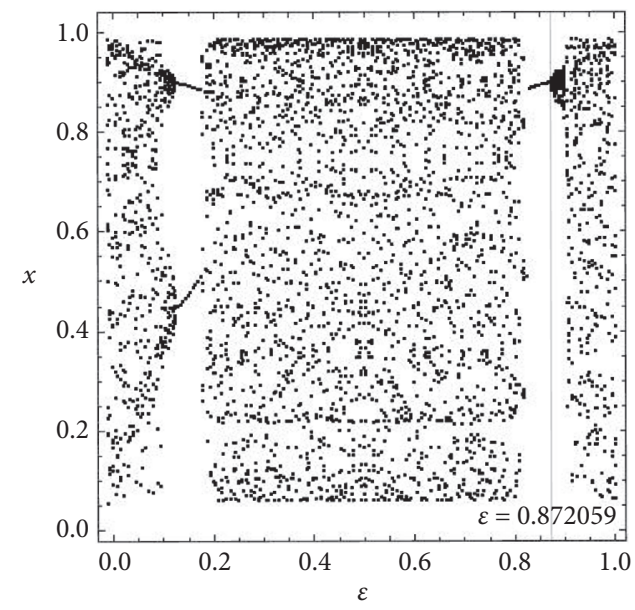

(a)

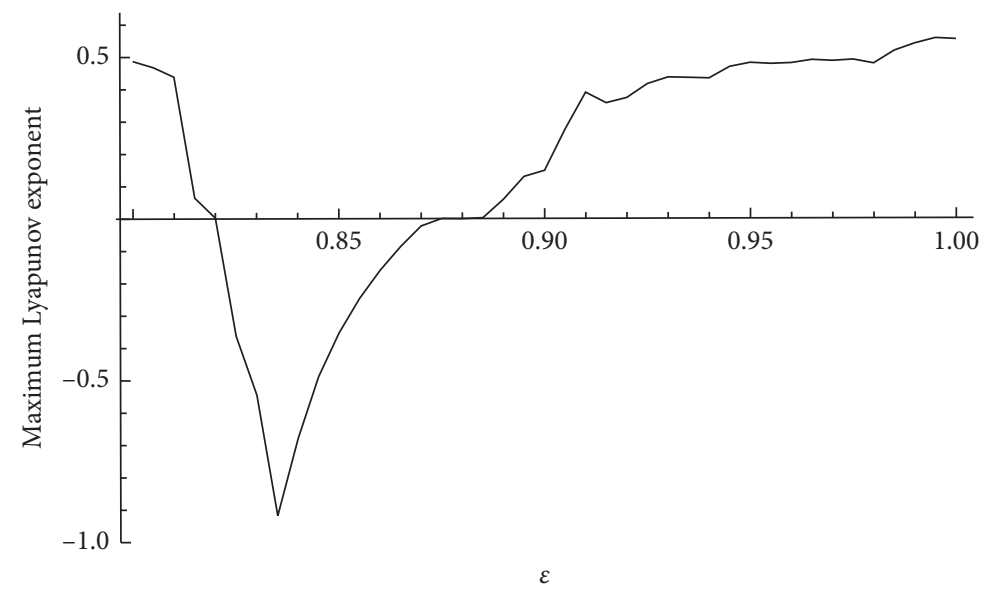

(b)

FIGURE 9: Bifurcation diagram and plot of the maximum Lyapunov exponent for $r=3.94$.

\section{Conclusion}

In this work, we investigated the dynamics of a discrete coupled system of logistic maps. We determined the stability of the system's fixed points and used center manifold and bifurcation theory to prove the existence of a flip and Neimark-Sacker bifurcation for the nonsymmetric fixed point $\left(x^{*}, y^{*}\right)$. Using $\epsilon$ as our bifurcation parameter, our numerical results indicate that the two-cycle is instrumental in establishing the flip bifurcation at $\left(x^{*}, y^{*}\right)$ above the critical $\epsilon$-value. This picture seems to hold for various $r$-values: for $r=3.1$, for instance, we see a flip bifurcation where the period is halved; for $r=3.83$, the intermittent twocycle in the chaotic regime appears to facilitate the transition to the stable one-cycle $\left(x^{*}, y^{*}\right)$.

For sufficiently large $r$-values, the one-cycle, initially established via the flip bifurcation, experiences a second bifurcation as $\epsilon$ increases where it loses stability. A general 
examination of the constant $a$ in Theorem 6 , as well as our numerical evidence, show that a Neimark-Sacker bifurcation occurs and is supercritical. Finally, we note that the rich dynamics of the system also includes interesting chaotic sets which will be analyzed further in a forthcoming work.

\section{Data Availability}

No data were used to support the findings of the study.

\section{Conflicts of Interest}

The authors declare that they have no conflicts of interest.

\section{References}

[1] M. Gyllenberg, G. Söderbacka, and S. Ericsson, "Does migration stabilize local population dynamics? Analysis of a discrete metapopulation model," Mathematical Biosciences, vol. 118, no. 1, pp. 25-49, 1993.

[2] B. E. Kendall and G. A. Fox, "Spatial structure, environmental heterogeneity, and population dynamics: analysis of the coupled logistic map," Theoretical Population Biology, vol. 54, no. 1, pp. 11-37, 1998.

[3] D. Fournier-Prunaret and R. Lopez-Ruiz, "Complex behaviour in a discrete coupled logistic model for the symbiotic interaction of two species," Mathematical Biosciences and Engineering, vol. 2, pp. 307-324, 2004.

[4] L. M. Saha and N. Sahni, "Chaotic evaluations in a modified coupled logistic type predator-prey model," Applied Mathematical Sciences, vol. 6, pp. 6927-6942, 2012.

[5] S. S. Askar, A. A. Karawia, A. Al-Khedhairi, and F. S. AlAmmar, "An algorithm of image encryption using logistic and two-dimensional chaotic economic maps," Entropy, vol. 21, pp. 1-17, 2019.

[6] A. A. Elsadany, A. M. Yousef, and A. Elsonbaty, "Further analytical bifurcation analysis and applications of coupled logistic maps," Applied Mathematics and Computation, vol. 338, pp. 314-336, 2018.

[7] H. Solis-Sanchez and E. G. Barrantes, "Using the logistic coupled map for public key cryptography under a distributed dynamics encryption scheme," Information, vol. 9, no. 160, pp. 1-12, 2018.

[8] L. Q. English and A. Mareno, "Symmetry breaking in symmetrically coupled logistic maps," European Journal of Physics, vol. 40, pp. 1-15, 2019.

[9] A. L'Her, P. Amil, N. Rubido, A. C. Marti, and C. Cabeza, "Electronically implemented coupled logistic maps," European Journal of Physics B, vol. 89, 2016.

[10] H. Mhiri, M. Tian, E. Wynne, S. Jones, A. Mareno, and L. Q. English, "An experimental survey of chaos and symmetry breaking in coupled and driven logistic maps," European Journal of Physics, vol. 40, pp. 1-14, 2019.

[11] T. K. R. Baishya, M. Chandra Das, and H. K. R. Sarmah, "Neimark Sacker bifurcation in delayed logistic map," International Journal of Applied Mathematics and Statistical Sciences, vol. 3, pp. 19-34, 2014.

[12] R. Paul and H. K. Sarmah, "Chaotic behavior in the coupled logistic map," International Journal of Advanced Scientific and Technical Research, vol. 6, pp. 337-357, 2012.

[13] J. Carr, Applications of Centre Manifold Theory, SpringerVerlag, New York, NY, USA, 1st edition, 1981.
[14] S. Wiggins, Introduction to Applied Nonlinear Dynamical Systems and Chaos, Springer Science, New York, NY, USA, 1990.

[15] J. Guckenheimer and P. Holmes, Nonlinear Oscillations, Dynamical Systems, and Bifurcations of Vector Fields, Springer-Verlag, New York, NY, USA, 1983.

[16] S. N. Elaydi, Discrete Chaos with Applications in Science and Engineering, Chapman and Hall/CRC, Boca Raton, FL, USA, 2nd edition, 2008. 